Article

\title{
A Novel Hybrid Search and Remove Strategy for Power Balance Wireless Charger Deployment in Wireless Rechargeable Sensor Networks
}

\author{
Tu-Liang Lin, Hong-Yi Chang * ${ }^{-}$and Yu-Hsin Wang \\ Department of Management Information System, National Chiayi University, Chiayi 60054, Taiwan; \\ tuliang@mail.ncyu.edu.tw (T.-L.L.); s1064629@mail.ncyu.edu.tw (Y.-H.W.) \\ * Correspondence: hychang@mail.ncyu.edu.tw
}

Received: 29 February 2020; Accepted: 20 May 2020; Published: 25 May 2020

\begin{abstract}
Conventional sensor nodes are often battery-powered, and battery power limits the overall lifetime of the wireless sensor networks (WSNs). Wireless charging technology can be implemented in WSNs to supply power to sensor nodes and resolve the problem of restricted battery power. This type of mixed network is called wireless rechargeable sensor networks (WRSNs). Therefore, wireless charger deployment is a crucial task in WRSNs. In this study, the method of placing wireless chargers to efficiently extend the lifetime of the WRSNs is addressed. Owing to the data forwarding effect in WSNs, sensor nodes that are closer to the data collection or sink node drain more power than nodes that are further away from the data collection or sink node. Therefore, this study proposes a novel hybrid search and removal strategy for the power balance charger deployment method. The wireless chargers are placed in the chosen nodes of the WRSNs. The node-chosen problem we address is called the dominating set problem. The proposed hybrid search and removal strategy attempts to discover the minimum number of chargers required to cover all sensor nodes in the WRSN. The proposed algorithm considers the charging power of the wireless directional charger when arranging its placement to maximize the charging capacity in a power-balanced prerequisite. Therefore, the proposed deployment strategy preserves the awareness of the presence of the sink node that could result in unbalanced power distribution in WRSNs. The simulation results show that the proposed strategy spares more chargers and achieves better energy efficiency than other deployment approaches.
\end{abstract}

Keywords: wireless rechargeable sensor networks; hybrid method; wireless charger deployment

\section{Introduction}

In a wireless sensor network (WSN), the amount of power provided by the battery determines whether each sensor can operate normally. Such power limitations also affect the stability of the entire WSN. To solve the problem of limited battery power, scholars have proposed studies on the design of energy-efficient routing protocols [1] and energy-harvesting wireless sensor networks (WSNs) to reduce energy consumption [2]. Neither the energy-efficient protocols nor the energy-harvesting WSNs solves the principle problem. These studies can prolong the life of WSNs. However, when the sensor exhausts the battery, the WSNs still cannot work properly. Some might argue that WSNs using solar energy can solve the problem of battery exhaustion. However, solar energy-based sensors are affected by environmental factors, and the efficiency of WSNs is significantly affected by different environments. In recent years, some researchers have proposed wireless charging technology, which can solve the problem of sensor battery power limitation, thus fundamentally solving the WSN lifetime problem [3]. The power can be transmitted using wireless chargers to RFIDs [4], sensors [5] and 
other terminal devices. TechNavio's market research shows that wireless charging technology has great market potential, and the 2020 global annual increase in the wireless charging market is more than $33 \%$ [6]. Due to the convenience and continuous development of wireless charging technology, wireless charging has drawn the attention of both industry and academia, especially in the realm of WSN research.

The adoption of wireless charging technology to improve the survival time of WSNs has been extensively surveyed. The new type of WSN that allows wireless chargers to extend the lifetime of wireless sensors is called wireless rechargeable sensor networks (WRSNs). The main consideration for the deployment of wireless chargers in WSNs is to maintain a stable power supply for the sensors so that the sensors can perform sensing tasks in the sensing area. Based on cost considerations, the number of wireless chargers in this deployment area should be minimized. However, because the power supply of the sensors has a significant impact on the performance of the WSNs, this study attempts to find a deployment strategy that can effectively reduce the number of wireless chargers while maximizing the charging capacity.

Several studies related to the deployment of wireless chargers in WRSNs have been proposed in recent years. However, these studies have some limitations, such as the grid partition that does not fit to the real environment in which the charger can be placed. Some might unrealistically assume that the data collection node or sink node does not exist. The data collection node or sink node in WSNs or WRSNs is a critical device. The sensors collect and route data to a data collection or sink node. If a sensor is near the data collection or sink node, it will bear frequent data forwarding and consume more energy. Therefore, to address the limitations of previous research, this study explores the deployment of directional chargers in a given network. In this study, the influence of data collection or sink nodes is evaluated. Previous studies have failed to address this influence. We also deal with two different types of chargers: omnidirectional and directional chargers in WRSNs. In this study, a two-stage strategy called search most and remove useless (SMRU) is proposed. In the first stage, the candidate position of the directional charger is selected on a sensor randomly. Next, we search for the number of sensors covered by the chargeable radius at the candidate position of the directional charger. The deployment given by the proposed SMRU strategy can effectively reduce the required number of directional chargers and preserve good charging utility. The experimental results demonstrate that our proposed SMRU approach outperforms previous works.

The three main contributions of the proposed SMRU algorithm are as follows:

1. The SMRU algorithm can deploy the chargers in the WRSN in less time, and the number of chargers required is relatively small.

2. When the sensor position moves, the SMRU algorithm takes less time to correct and deploy the charger positions.

3. The SMRU algorithm can calculate the energy consumption of the sink node. Under the premise of supplying enough power to the sink node, the mechanism of the SMRU algorithm can reduce the number of chargers used to charge the sink node without affecting the number of chargers that charge other sensors.

The structure of this study is as follows. In Section 2, this study investigates the relevant literature and explores the problems of some deployment strategies. In Section 3, the problem description is presented. Section 4 presents the details of the proposed algorithms. Section 5 explains the experimental results and give conclusions. 


\section{Related Works}

\subsection{Omnidirectional and Directional Chargers}

Previous studies assumed that omnidirectional chargers have the same charging capacity at the same distance $[7,8]$. When the distance increases, the received power decays, and when the threshold distance is finally reached, the received power becomes zero. The charging range of an omnidirectional charger is defined as the center of the charging range. The coverage area of the directional charger is a sector $[7,8]$. Devices from different manufacturers may have different ranges of charging angles. Due to the somewhat steady power coverage and low cost, directional chargers are frequently used in WRSNs.

As the mathematical formulation of a charger's charging power, previous researchers defined the charging formula as follows (1) [9-11]:

$$
W\left(\operatorname{dist}\left(a_{i}, s_{i}\right)\right)=\left\{\begin{array}{ll}
\frac{\alpha}{\left.\left(\operatorname{dist}\left(a_{i}, s_{i}\right)+\beta\right)\right)}, & \operatorname{dist}\left(a_{i}, s_{i}\right) \leq r \\
0, & \operatorname{dist}\left(a_{i}, s_{i}\right)>r
\end{array} .\right.
$$

In Equation (1), $\operatorname{dist}\left(a_{i}, s_{i}\right)$ is the Euclidean distance between charger $a_{i}$ and sensor $s_{i} \alpha=\frac{\xi W_{0} G_{G} G_{s}}{C_{W}}\left(\frac{\lambda}{4 \pi}\right)^{2}$, $\lambda$ is the wavelength, and $\xi$ is the utility of the rectifier. $G_{a}$ and $G_{s}$ are the antenna gains of the charger and the sensor, respectively. $W_{0}$ is the charger power and $\beta$ is the compensation parameter for short-distance transmission and $C_{w}$ in $\alpha$ is polarization mismatch loss [9]. When the range is larger than the charging radius $r$, the charging power is set to 0 .

\subsection{Wireless Charger and Sensor Deployment}

The deployment of wireless chargers has drawn much attention recently. Several studies have been conducted in this area. He et al. proposed an energy provisioning method for wireless rechargeable sensor networks [12]. The proposed method addressed omnidirectional wireless charger deployment, and the wireless chargers were deployed in the region of interest to supply sufficient charging power to support the operations in WRSNs. However, He et al. probe the region using a conventional triangular method. Chiu et al. proposed a mobility-aware charger deployment scheme for WRSNs [13]. The wireless charger placement methods proposed by Chiu et al. [13] considered the trajectories of mobile sensors. The methods partitioned the regions into grids, and omnidirectional wireless chargers were arranged on grid intersections. Liao et al. scheduled the sleep period of wireless chargers to reduce the wastage of battery power and adopted a conical structure to cover the region [7]. In contrast to studies on omnidirectional sensor deployment, some researchers have carried out studies on directional sensor deployment, which present the directional angle concept $[8,14]$. Horster and Lienhart discussed the placement of visual sensors that are directional, and the angles of the devices are considered [8]. According to Horster and Lienhart, the monitoring area of a visual sensor is directional and triangles are used to represent the scope [8]. Therefore, they proposed the integer linear programming solving technique to calculate the optimal coverage, and every grid intersection represented a possible site of the placement [8]. As the deployment of wireless chargers can be formulated as a nonlinear programming problem, the deployment problem befits to the NP hard problem class [15]. Therefore, solving the wireless charger deployment problem is a challenging task. Han et al. studied the deployment of directional sensors [14]. In their study, the number of deployed directional sensors was minimized, and the connections among the sensors were considered. Mo et al. addressed the coordination problem among multiple mobile chargers and formulated the multiple mobile charger coordination problem as mixed-integer linear programming and proposed a decomposition approach to solve the problem [16]. Tang et al. simultaneously considered both charging and routing and proposed an optimization approach to extend the lifetime of the network [17]. In order to balance the network energy, charging efficiency is dynamically balanced and the charging time is partitioned according to the energy consumption. Chen et al. proposed a WRSN model equipped 
charging drone [18]. A scheduling algorithm to solve the shortest multi-hop path is developed for the charging drone.

Most studies did not address the impact of unbalanced battery power distribution caused by data forwarding to the sink node. Unbalanced battery power distribution can make some sensor nodes, especially those near the sink node, lose power quicker than other nodes. Previous research did not consider the existence of a data collection node or sink node. Although the assumption can allow chargers in an actual environment to be better optimized for deployments, the deployments would actually result in an unbalanced battery power consumption issue. To address the unbalanced battery power distribution controversy, Lin et al. proposed a power balance aware deployment (PBAD) method for wireless chargers and compared the PBAD with random position and random orientation (RPRO) [10]. They formulated the deployment as a minimum dominating set problem and attempted to discover the minimal number of chargers required to cover the networks in the WRSNs. Lin et al. adopted a greedy algorithm to calculate the coverage set, and the influence of the data sink node was weighted to attain full coverage [10]. Lin et al. proposed a two-stage method. First, the chosen region was split into several sub-areas, so that each sub-area could have a continuous supply of charging power. Next, every sub-area was further evaluated to find the minimum dominating sets, after which an approximated optimal dominating set was chosen. The reviewed literature is tabulated in Table 1.

Table 1. Related deployment literature.

\begin{tabular}{|c|c|c|c|c|c|}
\hline Research & Sensor or Charger & Charging Type & & Important Concepts & Balanced Battery Power Distribution \\
\hline He et al., (2012) [12] & Charger & Omnidirectional & $\begin{array}{l}1 . \\
2 .\end{array}$ & $\begin{array}{l}\text { Energy provisioning } \\
\text { in WRSNs } \\
\text { Physical characteristics of } \\
\text { wireless charging }\end{array}$ & No \\
\hline $\begin{array}{l}\text { Chiu et al., } \\
\text { (2012) [13] }\end{array}$ & Charger & Omnidirectional & $\begin{array}{l}1 . \\
2 .\end{array}$ & $\begin{array}{l}\text { Mobility-Aware } \\
\text { charger deployment } \\
\text { Optimization of } \\
\text { mobile deployment }\end{array}$ & No \\
\hline $\begin{array}{l}\text { Liao et al., } \\
\text { (2013) [7] }\end{array}$ & Charger & Omnidirectional & $\begin{array}{l}1 . \\
2 .\end{array}$ & $\begin{array}{l}\text { Optimized charger } \\
\text { deployment for WRSNs } \\
\text { Sleep scheduling }\end{array}$ & No \\
\hline $\begin{array}{c}\text { Horster and } \\
\text { Lienhart., (2006) [8] }\end{array}$ & Sensor & Directional & $\begin{array}{l}1 . \\
2 .\end{array}$ & $\begin{array}{l}\text { Approximating optimal } \\
\text { visual sensor placement } \\
\text { Grid linear programming }\end{array}$ & No \\
\hline $\begin{array}{l}\text { Han et al., } \\
\text { (2008) [14] }\end{array}$ & Sensor & Directional & $\begin{array}{l}1 . \\
2 .\end{array}$ & $\begin{array}{l}\text { Deploying directional } \\
\text { sensor networks } \\
\text { Guaranteed connectivity } \\
\text { and coverage }\end{array}$ & No \\
\hline $\begin{array}{l}\text { Lin et al., } \\
\text { (2016) [10] }\end{array}$ & Charger & $\begin{array}{l}\text { Directional and } \\
\text { Omnidirectional }\end{array}$ & $\begin{array}{l}1 . \\
2 .\end{array}$ & $\begin{array}{l}\text { Power balance aware } \\
\text { wireless } \\
\text { charger deployment } \\
\text { Minimum dominating } \\
\text { set problem }\end{array}$ & Yes \\
\hline Mo et al. (2019) [16] & Charger & Omnidirectional & $\begin{array}{l}1 . \\
2 .\end{array}$ & $\begin{array}{l}\text { The coordination problem } \\
\text { among multiple } \\
\text { mobile chargers } \\
\text { Mixed-integer linear } \\
\text { programming problem }\end{array}$ & No \\
\hline $\begin{array}{l}\text { Tang et al., } \\
\text { (2020) [17] }\end{array}$ & Charger & Omnidirectional & $\begin{array}{l}1 . \\
2 .\end{array}$ & $\begin{array}{l}\text { Considering both charging } \\
\text { and routing } \\
\text { Partitioned charging time } \\
\text { according to the } \\
\text { energy consumption }\end{array}$ & Yes \\
\hline $\begin{array}{l}\text { Chen et al., } \\
\text { (2020) [18] }\end{array}$ & Charger & Drone & $\begin{array}{l}1 . \\
2 .\end{array}$ & $\begin{array}{l}\text { A WRSN model equipped } \\
\text { charging drone } \\
\text { The shortest multi-hop } \\
\text { path problem }\end{array}$ & No \\
\hline
\end{tabular}




\section{Search Most and Remove Useless Algorithm}

\subsection{Problem Definition}

There are several problems with wireless sensor network applications. Among them, the power and cost of sensors are often the focus of research. In a wireless sensing network, the sensor must operate continually to maintain the function of receiving and transmitting the information. Therefore, reducing the sensor's power consumption and extending battery life is an important problem. The network architecture called wireless rechargeable sensor network (WRSN) relies on the chargers to continuously supply the sensors with continuous power. In far-field phased arrays, wireless power can be transmitted through beams, while power beam transmission technology can transmit energy over longer distances. The wireless power transfer method proposed in the simulation design in this research is far-field phased array antennas [19]. As the deployment of wireless sensing networks may use a large number of sensors, the use of fewer chargers in WRSNs to provide sufficient power for the sensors to reduce deployment costs and maintain the operation of WRSNs is an important issue. Therefore, this study proposes a method that uses fewer directional chargers to cover all sensors in WRSNs. In our proposed method, the deployment position of the charger can be calculated in a short time. Additionally, all sensors in WRSNs are covered with a small number of chargers, and all sensor power is supplemented to avoid sensor interruption due to power consumption causing WRSNs to fail, and to reduce the cost of deploying a WRSN.

\subsection{The SMRU Algorithm}

The algorithm is divided into two parts. In the first part, the candidate position of the directional charger is selected on a sensor randomly. Next, we search for the number of sensors covered by the chargeable radius at the candidate position of the directional charger. The candidate position rotates $360^{\circ}$ to find the angle that can cover most of the sensors within the charging range. If the number of sensors covered by the candidate position of the directional charger is more than three, the algorithm is repeated for each candidate position within the chargeable radius. To find the angle that can cover most of the sensors within the charging range, the position that covers the most sensors is selected as the new position of the directional charger. If the number of sensors covered by the candidate position of the directional charger is less than three, the candidate position is determined as the position of the directional charger. In the second part, after determining the position of the directional charger, the proposed algorithm checks whether there is an unnecessary position of the directional charger. Therefore, the algorithm checks and removes the charger, and the sensors covered by the charger can be covered by other chargers. This check action can effectively reduce the number of chargers. The operation of SMRU represented as Algorithm 1 is as follows:

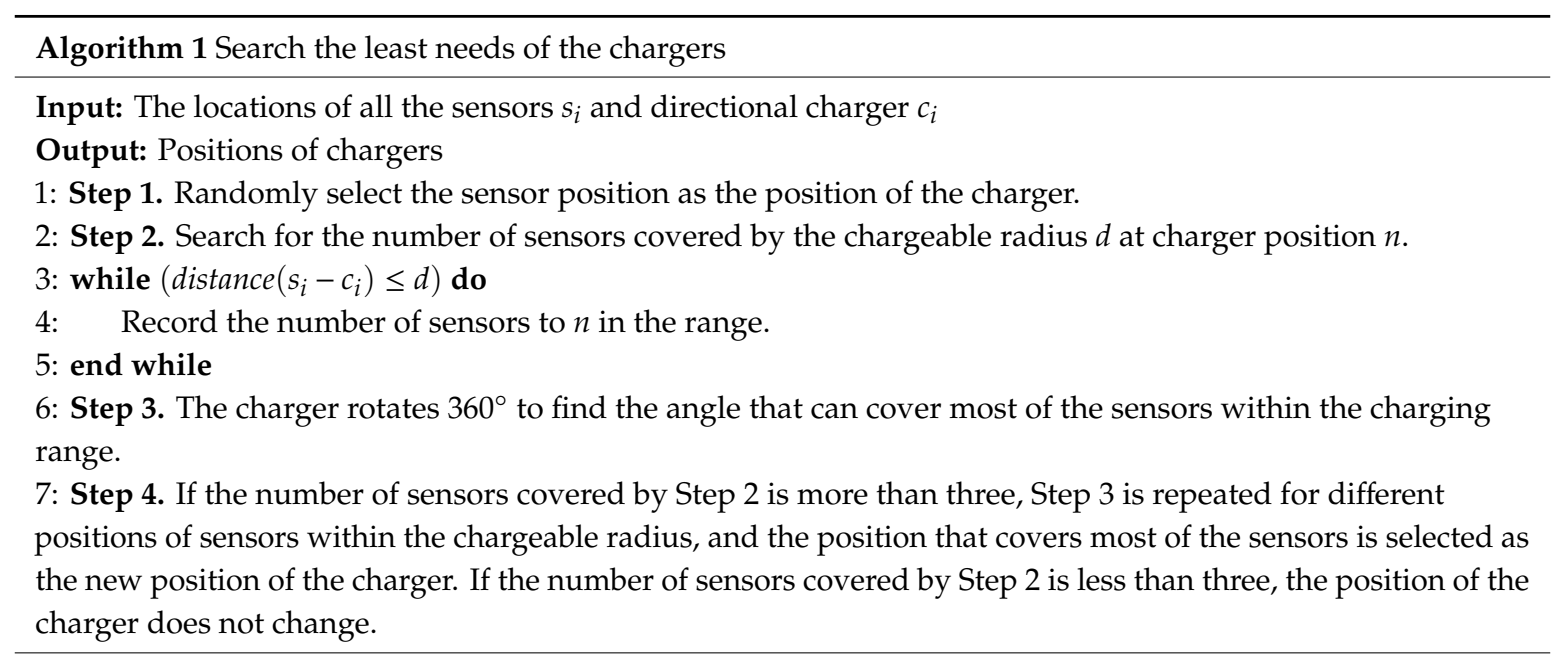




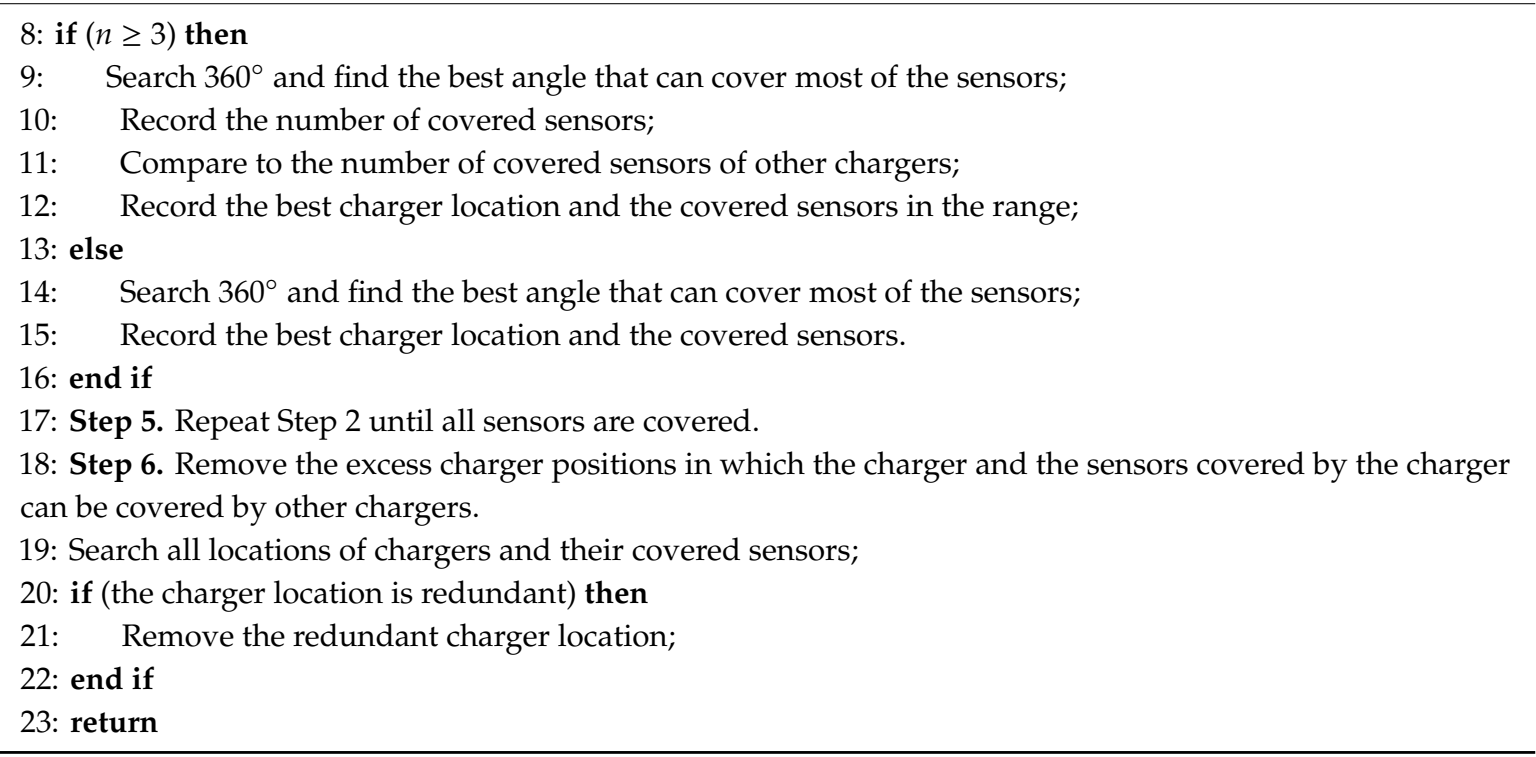

\subsection{An Example of SMRU}

In this study, we use the sensor position to deploy a charger, which can ensure that the worst result of the position selection will converge to the number of sensors. This means that when there are $\mathrm{N}$ sensors in WRSNs, it is only necessary to deploy $\mathrm{N}$ chargers in the worst case. This can prevent the position selection algorithm from converging. The first idea at the beginning of this study is to search for the charger position that first covers the most sensors among all sensor positions. However, this method is time-consuming because the same calculation is repeated at $360^{\circ}$ angles of the charger for each position. Therefore, this study improves the first idea. This study found that the key point to covering all sensors in WRSNs with fewer chargers is the charger position with dense sensors. For example, under the same sensor position configuration, if the charger position is selected as shown in Figure 1, the charge angle of the charger is limited, so that two chargers are required to fully cover all the sensors in this area. However, if the algorithm first compares the positions in the dense area and finds the position that can cover most of the sensors, the algorithm can reduce the number of chargers to one, as shown in Figure 2.

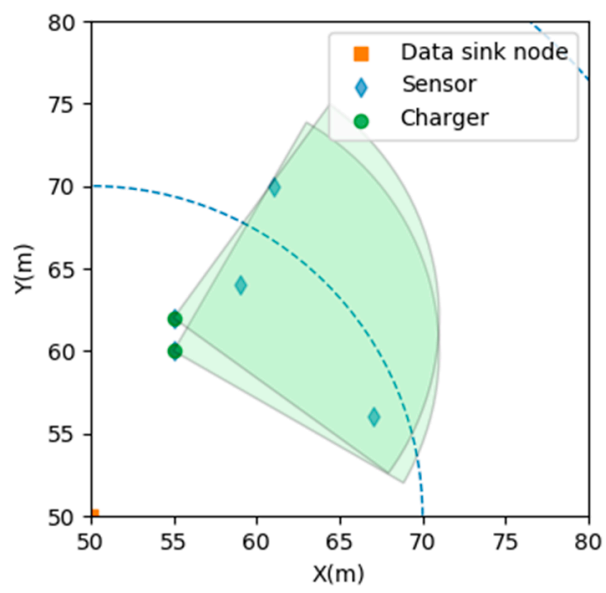

Figure 1. Covers all sensors with two chargers. 


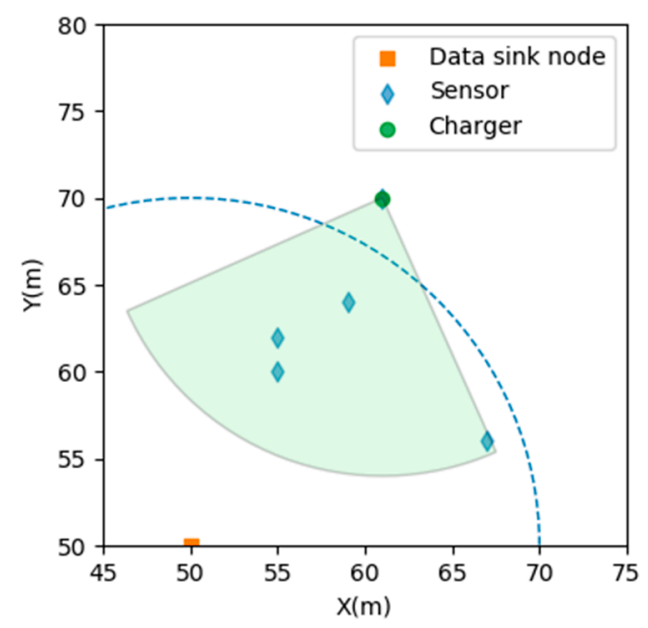

Figure 2. Covers all sensors with one charger.

Therefore, the proposed algorithm first randomly assigns the position of the charger and then checks to see if there are more than three sensors in the $360^{\circ}$ charging range of the charger, after which it selects the charger position that covers most of the sensors. If not, then directly select this position and find the best charging angle of the position to save the waste of repeated calculation time caused by the first idea. The reason for choosing more than three sensors in the charging range as the comparison standard is that if there are only one or two sensors that can be covered in the charging range, regardless of the selected position, the number of chargers in the charging range will not be affected. However, if the number of sensors that can be covered in the charging range is more than three, the results may be affected. As shown in Figure 3, if the algorithm selects the middle position, all sensors require two chargers. However, if the algorithm selects the two surrounding positions, it only needs one charger, as shown in Figure 4.

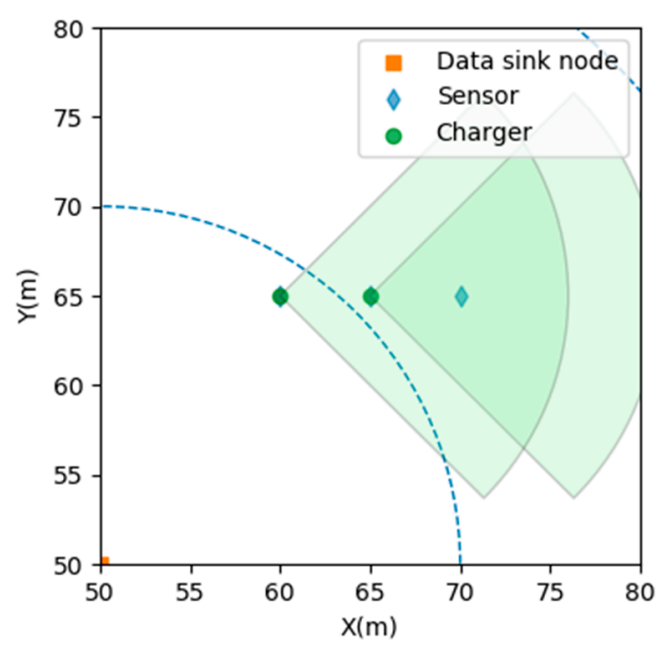

Figure 3. Covers all sensors with two chargers. 


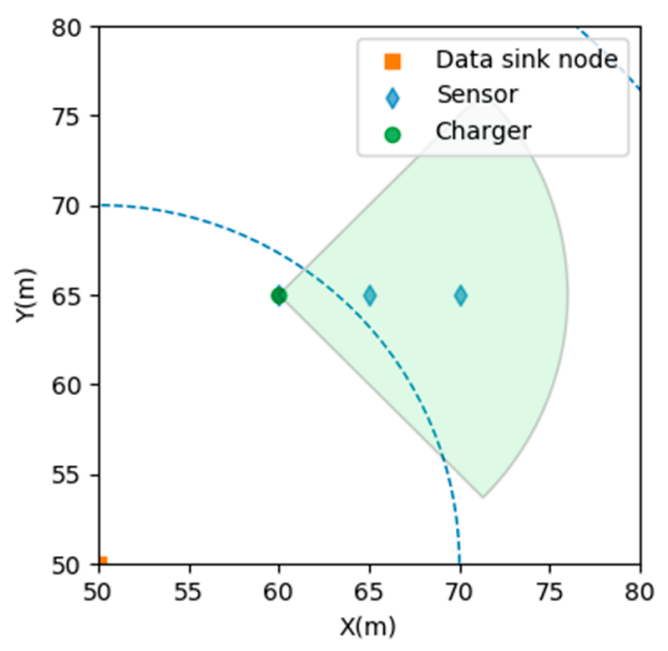

Figure 4. Covers all sensors with one charger.

Finally, although the proposed algorithm minimizes the number of chargers required, experimental results show that extra chargers are required in some cases. The main reasons are the random selection of positions as a sequence of charger deployment, the comparison of the other candidate positions in the charging coverage area of the charger, and the selection of the best position from the candidate positions. As shown in Figure 5, if there are sensors that are arranged continuously, but the distance exceeds the chargeable radius, it cannot be applied to the proposed algorithm. Assume that there are four consecutive sensor positions arranged horizontally. If the order of the positions of the chargers is not appropriate, three chargers are required to fully cover all the sensors in this area. Therefore, this study improves the proposed algorithm again. After the initial selection of the charger position, it is necessary to check whether each charger and the sensors covered by each charger can be covered by other chargers. Assuming that the position of the charger fits this context, it means that the position of this charger is redundant. Therefore, after checking, remove the excess charger positions, as shown in Figure 6.

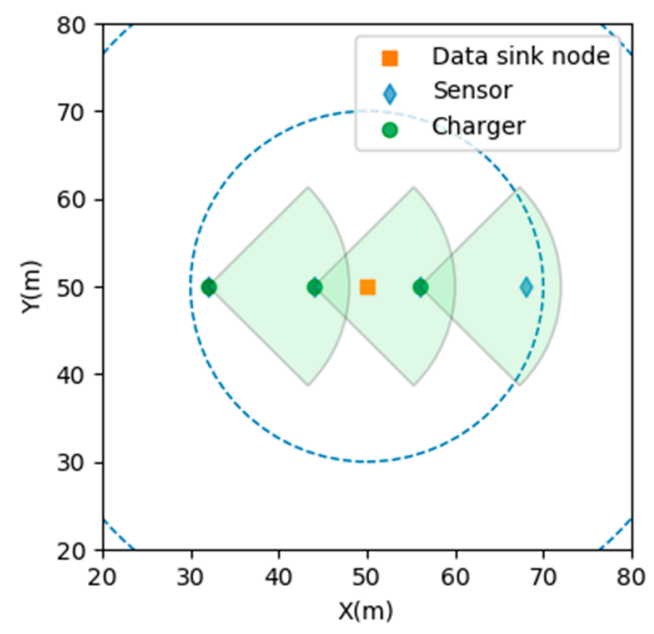

Figure 5. The position of this charger is redundant. 


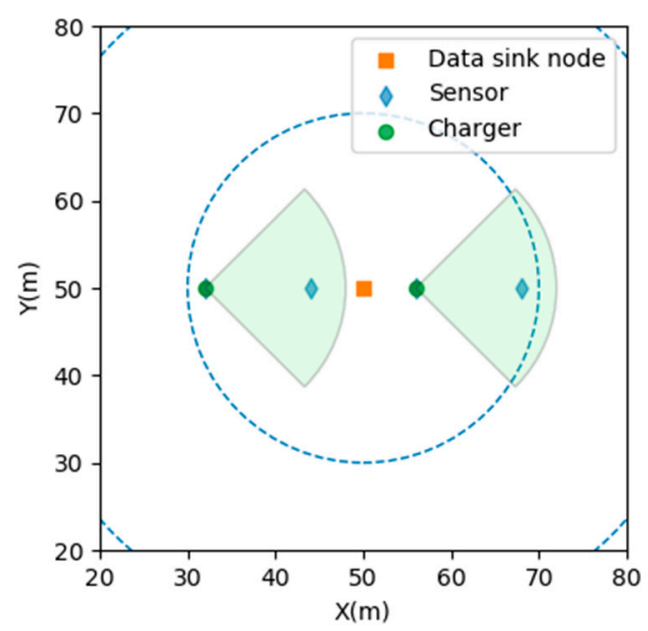

Figure 6. Remove the excess charger position.

\subsection{Sensor Mobility}

In this study, we consider how the SMRU algorithm can change the position of chargers when the position of the sensors is moved. After the sensor moves the SMRU algorithm first confirms whether the number of sensors covered by the charger has changed. If the number of covered sensors decreases, the charger is rotated $360^{\circ}$ to find the charging angle that can cover most of the sensors. If the number of covered sensors is unchanged or increased, the sensor data will be updated. Next, the SMRU algorithm examines whether there are any sensors in the WRSNs that are not being charged by any charger. If it is found that there are uncharged sensors, increase the chargers at the position of this sensor, and then rotate $360^{\circ}$ to find the best angle that covers the largest number of sensors. Finally, the SMRU algorithm performs an optimization operation. If it finds an excess charger, it cancels the deployment at that position. The sensor movement was not considered in the PBAD algorithm. As PBAD does not have a mechanism for sensor movement, it needs to be recalculated when the sensors are moved, which takes twice as much time. However, the SMRU algorithm only needs to modify the position of chargers that are affected by sensor movement. Therefore, the modified execution time is much shorter than the original SMRU execution time. The algorithm is shown in Algorithm 2.

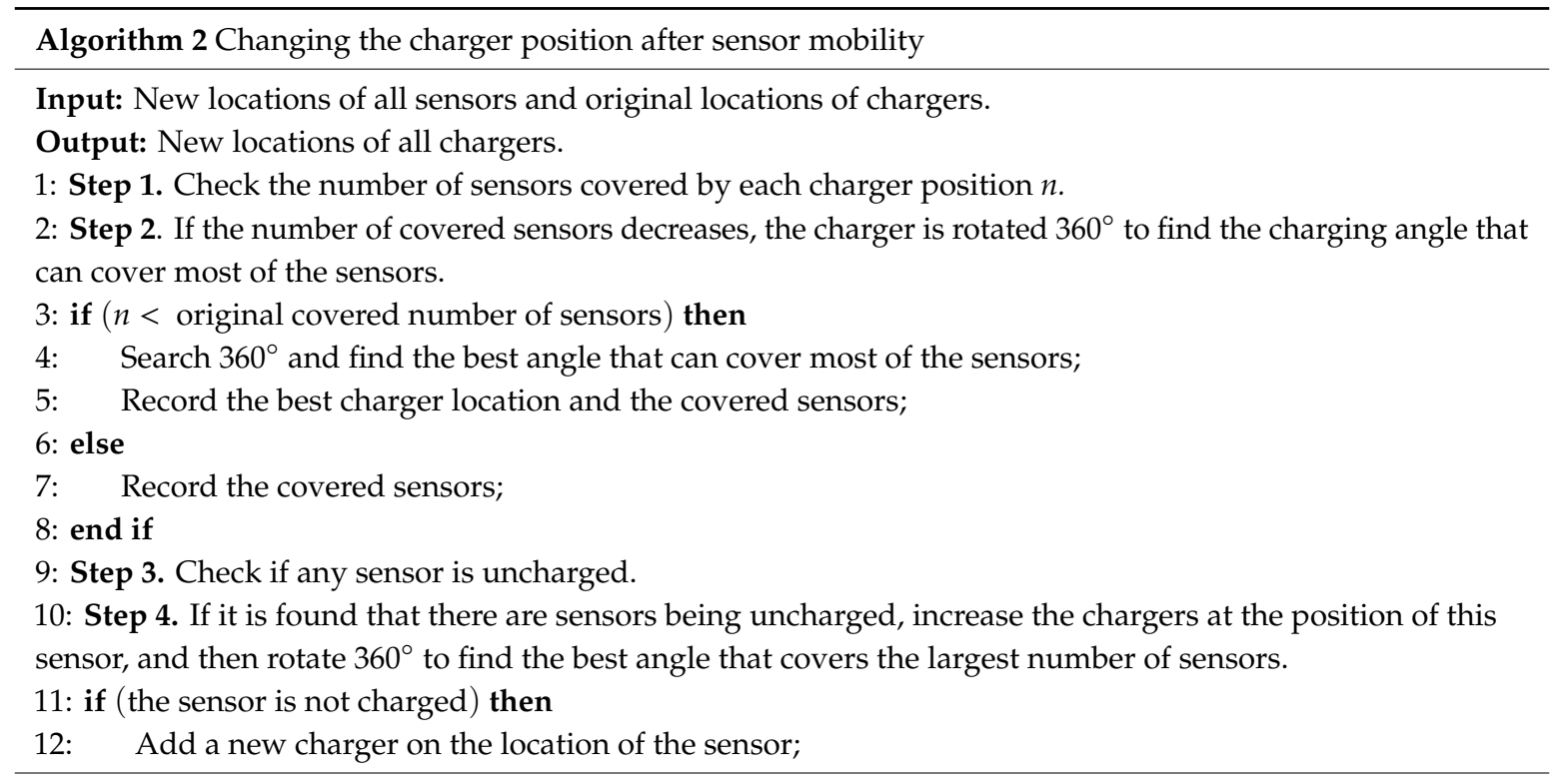




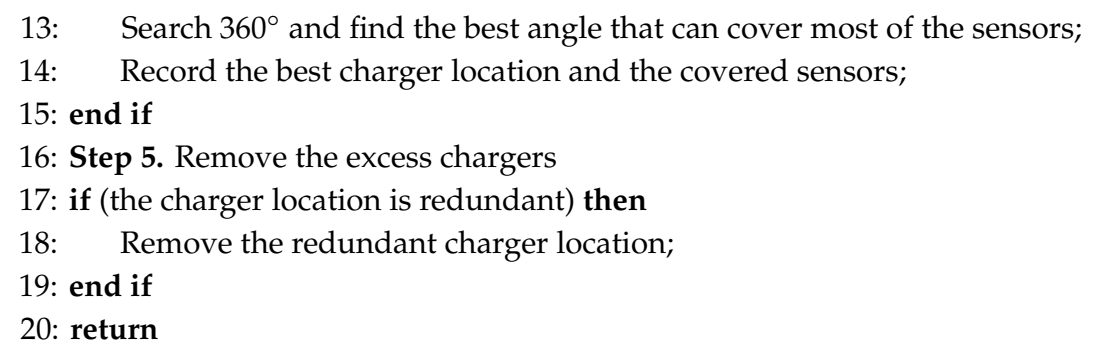

\subsection{The Charging of Sink Nodes}

The SMRU algorithm can calculate the energy consumption of the sink nodes. Under the premise of supplying enough energy to the sink nodes, the SMRU algorithm can reduce the number of chargers deployed to charge sink nodes. It also does not affect the number of chargers that need to charge sensors. First, the sensor will transfer the data to the sink that is closest to itself. After the SMRU has deployed the charger, it checks the amount of energy consumed by each sink. Next, the SMRU checks the charger within the charging distance of the sink and checks the number of sensors charged by the charger. If the number is equal to one, the charging angle is turned to sink. Therefore, the charger will charge one sensor and one sink. If the number of charging sensors is greater than 1 , the charger rotates $360^{\circ}$ again to search for the best angle, and the calculation includes the sink node. If the charging target can be covered more than the previous number (meaning that the previously charged sensors are covered; it also covers the sink node), the charging angle is updated; otherwise, the charger maintains the original charging angle. Finally, according to the energy that the sink still lacks, the SMRU deploys additional chargers around the sink to charge the sink. The number of chargers deployed can supplement enough energy as the minimum requirement. Consequently, all sink nodes can have enough power to receive the data sent by the sensor. The SMRU can make good use of the existing charger resources to charge the sink, so it can reduce the number of additional charger deployments. The algorithm is shown in Algorithm 3.

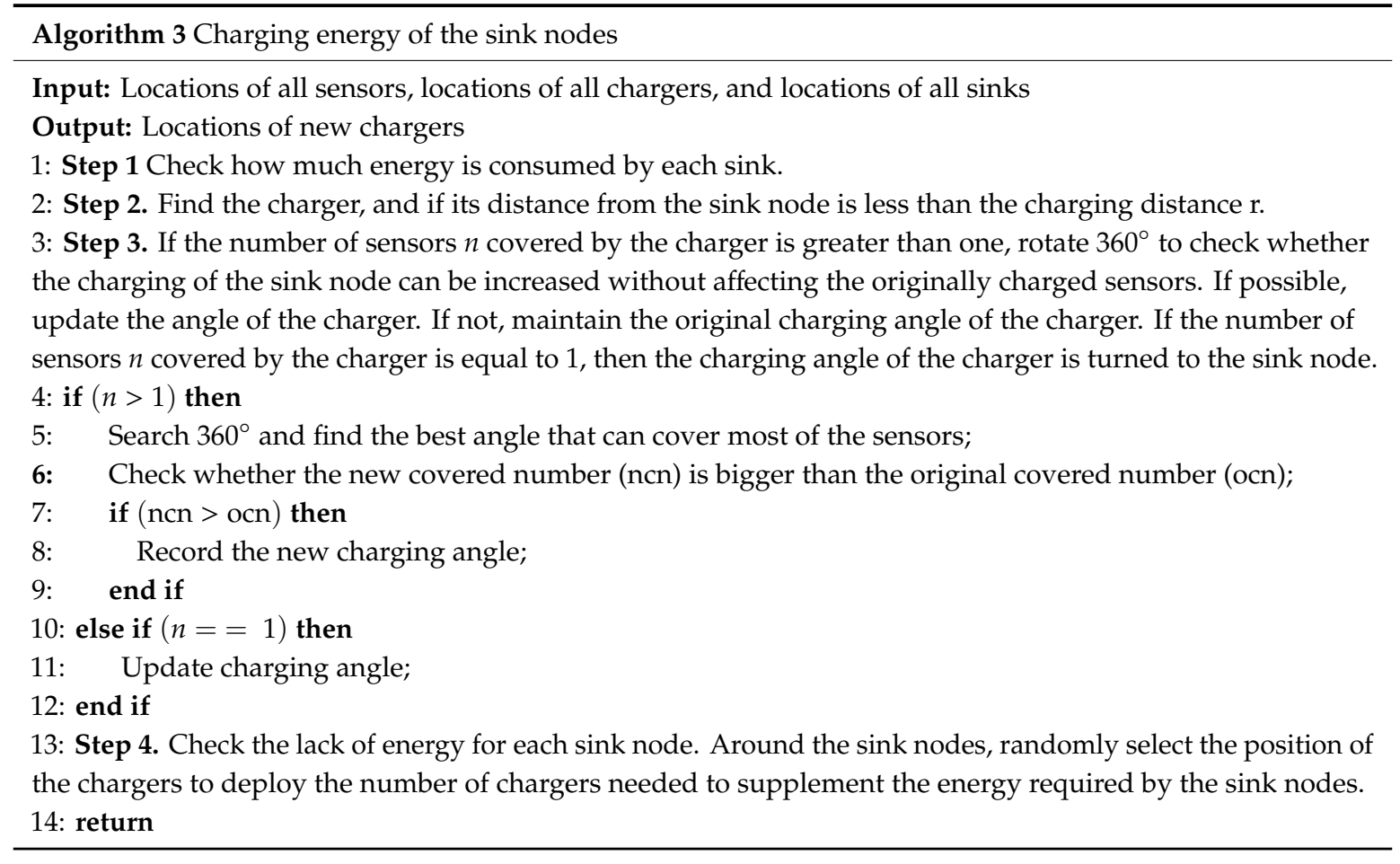




\section{Experiments and Results}

\subsection{Experimental Environment and Parameters}

In this study, a directional charger was used to conduct a simulation experiment. The experimental environment was to randomly generate sensor positions on a plane with a length of 100 units and a width of 100 units. The proposed algorithm was repeated 500 times to find the average result, and obtain the number of chargers and average execution time. As this study uses a directional charger, its related parameters are set to a chargeable angle of $90^{\circ}$ and a chargeable radius of 16 units. Assume that the reception energy consumption of the sink node is 0.6 units/reception. The performance of the proposed algorithm is compared with that of the random position and random orientation (RPRO) and power balance aware deployment (PBAD) [10] algorithms using directional chargers.

\subsection{Experimental Results of the Number of Chargers}

This experiment compares the relationship between the number of sensors, different algorithms, and the number of chargers required. The average number of chargers used by the SMRU algorithm is less than that used by RPRO and PBAD. Figure 7 shows the average number of chargers used. RPRO selects the charger position and the charging direction randomly. As the charging angle is not selected, the number of chargers required is significantly large. PBAD searches for $360^{\circ}$ angles that can cover most of the sensors for charging after randomly selecting positions. Additionally, PBAD repeatedly calculates the same sensor position map and selects the best position using a minimal charger as the deployment location of the charger. In this experiment, PBAD was repeated 10 times to select the best result. Therefore, the experimental results of PBAD are superior to those of RPRO. SMRU first selects a random position and then searches for $360^{\circ}$ of the charging radius at that position. If there are more than three sensors (three candidate positions) within the charging radius at this position, it chooses the candidate position that can cover the largest number of sensors and deploy the charger. Additionally, the elimination of the excess charger positions that may be generated in the previous steps means that the number of charger requirements can be reduced to less than that of PBAD.

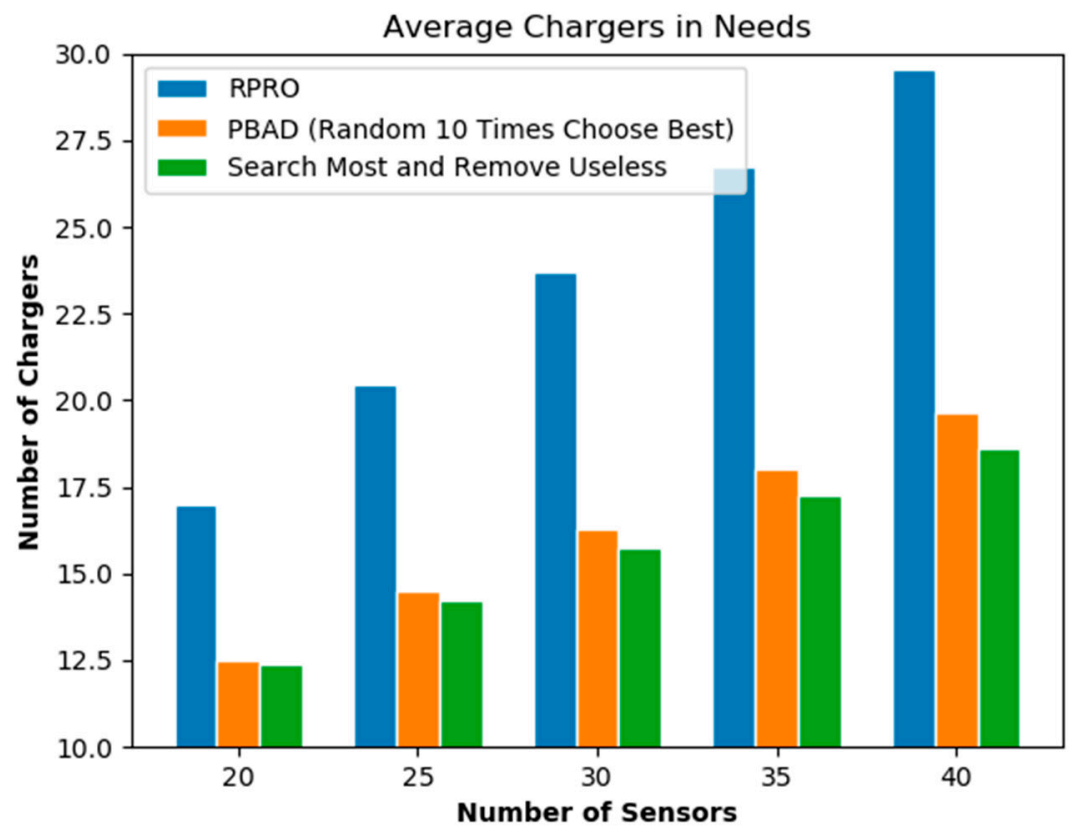

Figure 7. The average number of chargers. 


\subsection{Experimental Results of Execution Time}

This experiment compares the relationship between the execution time of different algorithms and the number of sensors. Figure 8 shows the average execution time. As the RPRO algorithm selects the position and charging direction of the charger randomly and does not have too many calculations, it requires little execution time. However, the number of chargers required by the RPRO algorithm is very large. The PBAD algorithm randomly selects the charger position and repeats the operation multiple times to obtain the best results. Although it can obtain good results, the iterative operations are performed multiple times. As a result, the average execution time is more than that of SMRU and RPRO, and the execution time will increase as the number of recalculations increases. When SMRU selects the position that covers the largest sensor, it decides whether to compare multiple positions. Only when the number of sensors (candidate positions) around the position is more than three will it be executed. This is because when the number of candidate positions is less than three, regardless of the position selected, it does not affect the coverage results, and thus can reduce the execution time of many repeated calculations. Additionally, the SMRU algorithm only needs to be executed once to obtain the results, and it is not necessary to repeatedly take the best value like PBAD. Therefore, the execution time performance is much better than that of PBAD.

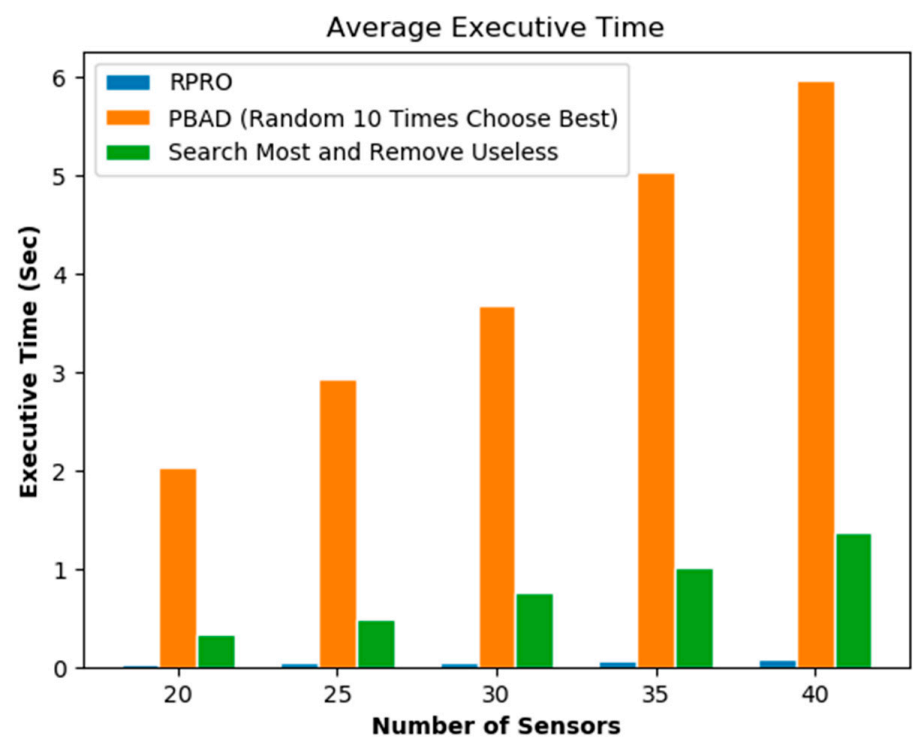

Figure 8. The average execution time.

\subsection{Experimental Results for Different Charging Angles}

This experiment compares the relationship between the size of the charging angle and the average number of chargers. The experimental angle ranges from $45^{\circ}$ to $360^{\circ}$. The RPRO, PBAD, and SMRU algorithms were tested separately. The charging radius is 16 units, and the number of sensors is 40 . The experiment found that the larger the charging angle, the fewer the number of chargers required. The different charger requirements for different algorithms are as follows: SMRU has the smallest, followed by PBAD, and the worst is RPRO. Additionally, for the same parameter settings of the same algorithm, there seems to be a lower demand limit on the number of charger requirements. As shown in Figure 9, it can be observed from the experimental results that there is no significant difference in the average number of chargers after $270^{\circ}$ between PBAD and SMRU. However, the difference is significant when the angle is smaller than $270^{\circ}$. 


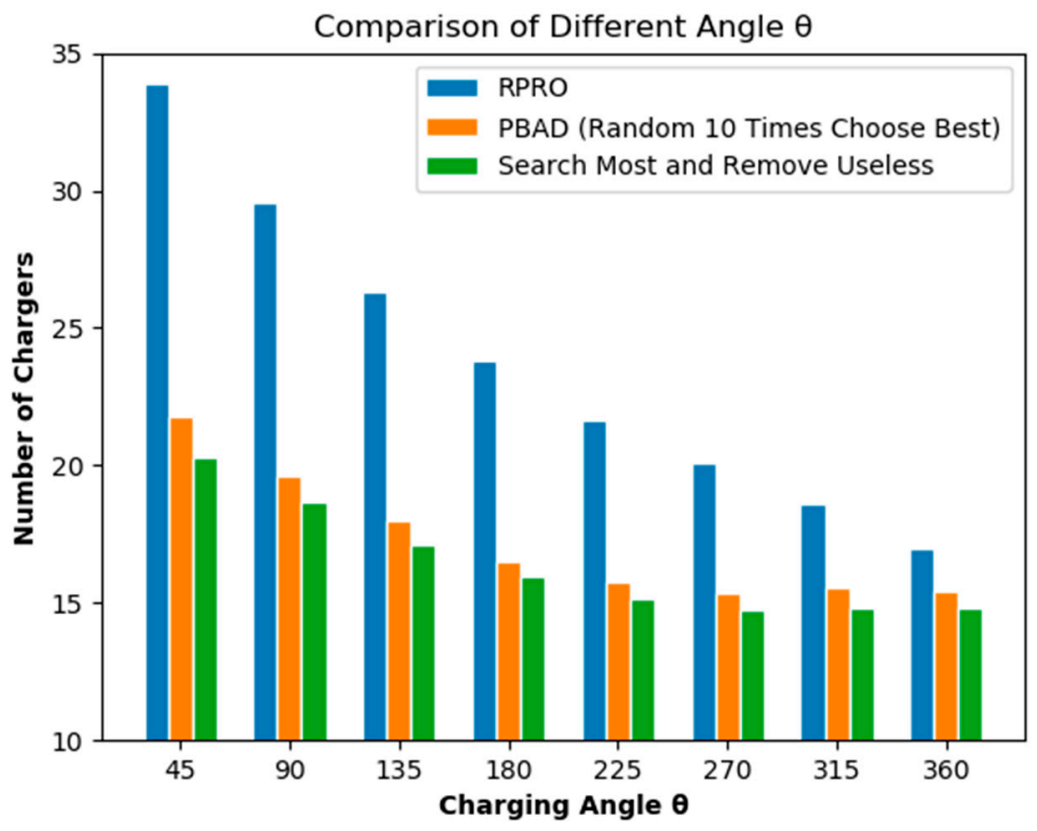

Figure 9. The comparison results of different charging angles.

\subsection{The Comparison of the Execution Time of Charger Deployment and Modified Deployment Using SMRU}

In the experiment, we measured the execution time it takes for the SMUR algorithm to adjust the position of chargers after sensor mobility. After the SMRU selects the charger position, this experiment randomly moves the sensors from 0 to 2 units to simulate the movement of the sensors. A comparison of the execution time of charger deployment and modified deployment using SMRU is shown in Figure 10.

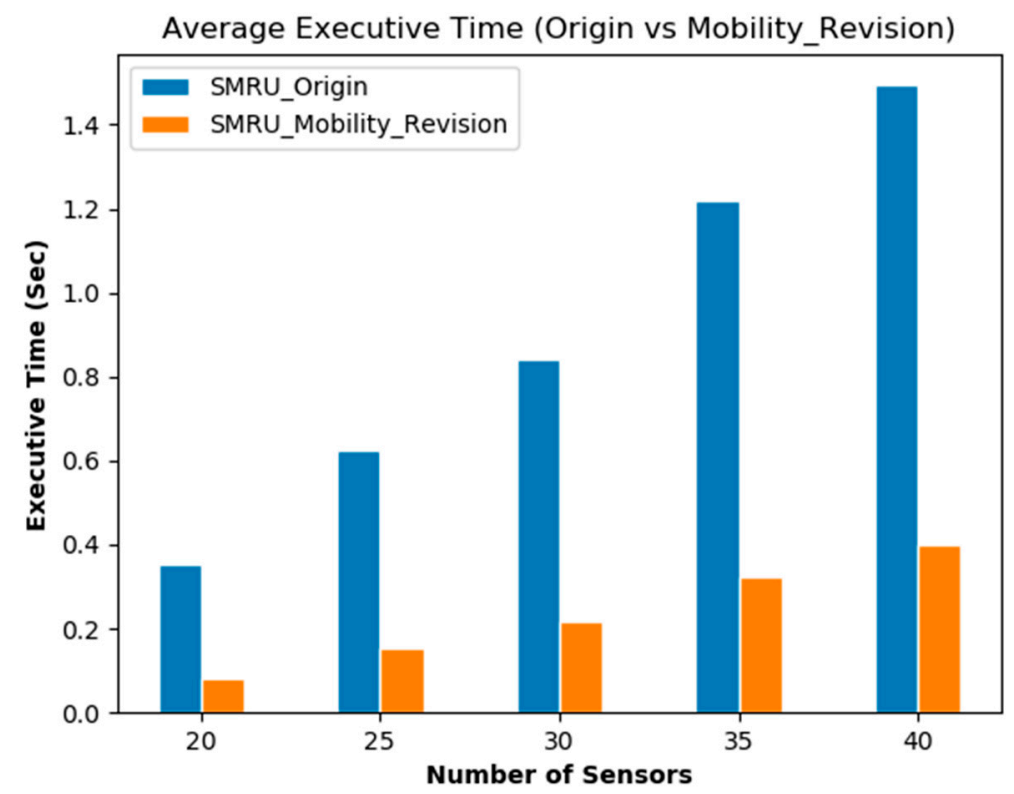

Figure 10. Comparison of the execution time of charger deployment and modified deployment using SMRU.

\subsection{The Impact of Different Mobility Units}

In this experiment, we first discuss the impact of different mobility units and average execution time for SMRU to adjust the position of chargers after sensor mobility. After the SMRU selects the charger position, this experiment randomly moves sensors from 2 to 10 units to simulate the movement 
of sensors. Due to SMRU does not recalculate the positions of all the chargers to find the global optimal solution after sensor mobility; it can reduce many execution times. The experimental results show that the execution time will increase with the number of sensors, and the increase in the mobility units of the sensor will increase the average execution time, as shown in Figure 11.

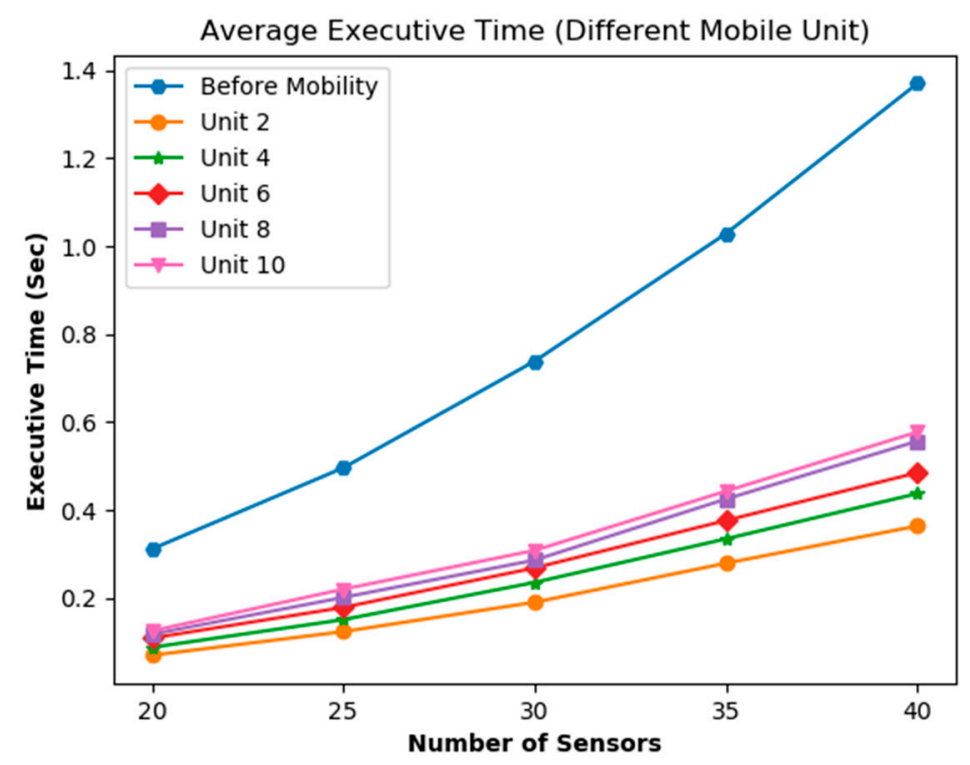

Figure 11. The impact of different mobility units and average execution time for search most and remove useless (SMRU) strategy to adjust the position of chargers after sensor mobility.

Next, we discuss the number of chargers used for SMRU to adjust the position of chargers after sensor mobility. Although SMRU does not recalculate the positions of all the chargers to find the global optimal solution after sensor mobility. However, the experimental results found that the number of chargers used does not increase too much after the sensor mobility. In addition, the number of chargers will increase with the number of sensors, and there is no obvious difference in the number of chargers used between different mobility units, as shown in Figure 12 .

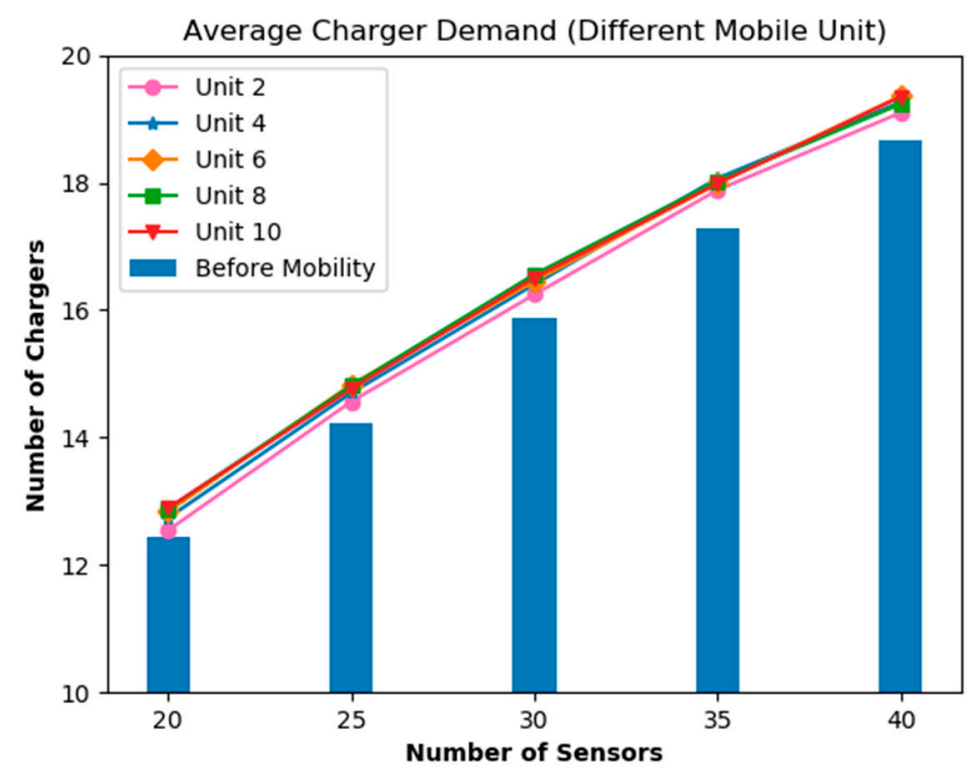

Figure 12. The impact of different mobility units and the number of chargers used for SMRU to adjust the position of chargers after sensor mobility. 


\subsection{Energy Consumption and Supplementary of Sink Nodes with Different Sink Rates}

In this experiment, we discuss how to deploy enough chargers with different sink rates. In the experiment, an average of four sink nodes (SN) were deployed in a $100 \times 100$ unit experimental environment, with coordinates SN_1 $(25,25)$, SN_2 $(25,75)$, SN_3 $(75,25)$, SN_4 $(75,75)$. As all sensors need to send data to the sink node, it is assumed that the radius of the received data of the sink is 36 units in the experiment to cover the entire experimental environment. Every time a sensor transmits data to the sink node, it increases the sink energy consumption by 0.6 units. Each charger can charge 1 unit of energy to the sink. The total number of sensors deployed in the experimental environment was 20. This experiment is based on being able to replenish all the energy needed by the sink nodes, and deploying enough chargers around the sink node to replenish the power. Figure 13 shows the energy consumption and supplementary of sink nodes with different sink rates.

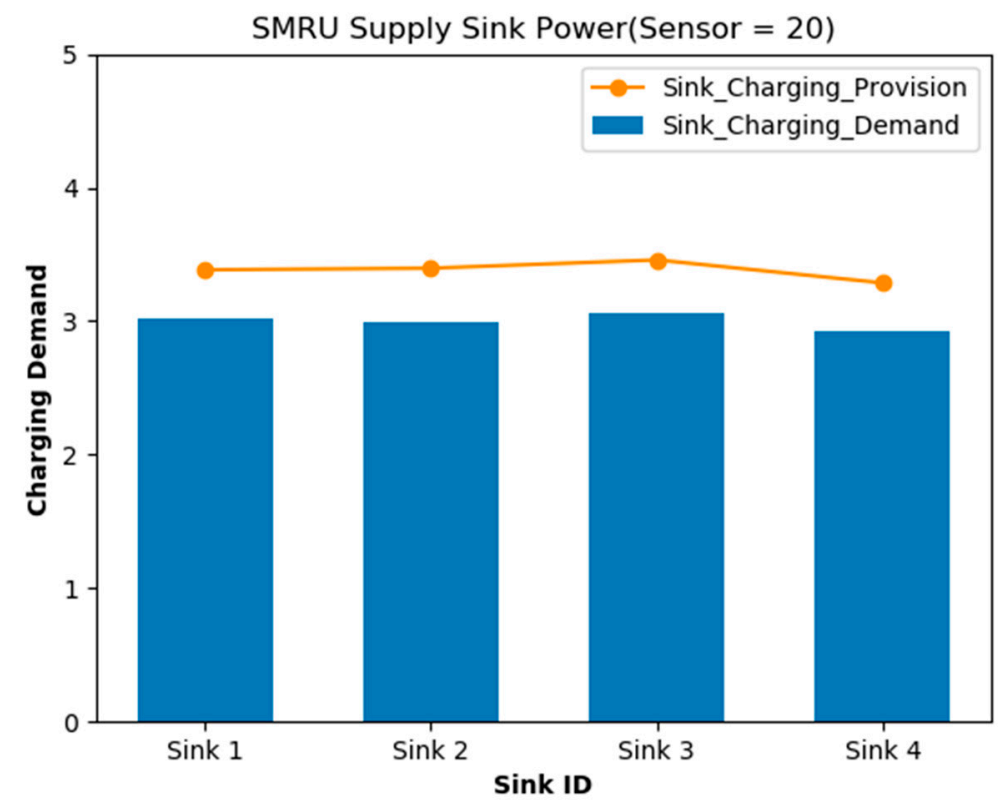

Figure 13. The energy consumption and supplementary of sink nodes with different sink rates.

\subsection{The Impact of Different Densities on the Energy Requirements of Sink Nodes}

This experiment compares the impact of different densities on the energy requirements of sink nodes, as shown in Figure 14. This experiment deploys four kinds of sink node density. The first case is $4 \operatorname{COS}(\mathrm{C}=\mathrm{Close}, \mathrm{S}=$ Separate $)$ representing four sink nodes that have overlapping coverage. The second case is $2 \mathrm{C} 2 \mathrm{~S}$ representing two sink nodes that have overlapping coverage (Sink 1 , Sink 2) and two isolated sink nodes (Sink 3, Sink 4). The third case is 3C1S represents three sink nodes that have overlapping coverage (Sink 1,2,3) and one isolated sink node (Sink 4). The fourth case is 0C4S, which means four sink nodes are isolated. The positions of sensor nodes are randomly deployed in the coverage of sink nodes. The experimental results found that the average energy requirements of sink nodes is almost the same in 0C4S and 4COS situation, as shown in Figure 12. In the case of sink nodes that have overlapping coverage, sink nodes can share the processing of sensor nodes in the same coverage, and so the energy requirements of sink nodes will be shared and reduced. Therefore, the average energy requirements of sink nodes are the same as the four isolated sink nodes. In $2 \mathrm{C} 2 \mathrm{~S}$ and 3C1S situation, some sink nodes have overlapping coverage and some sink nodes are isolated. As isolated sink nodes have no other sink nodes in the coverage to help process the data from sensor nodes in the coverage, this causes an increase in the energy requirements of sink nodes, as shown in Figure 14. 


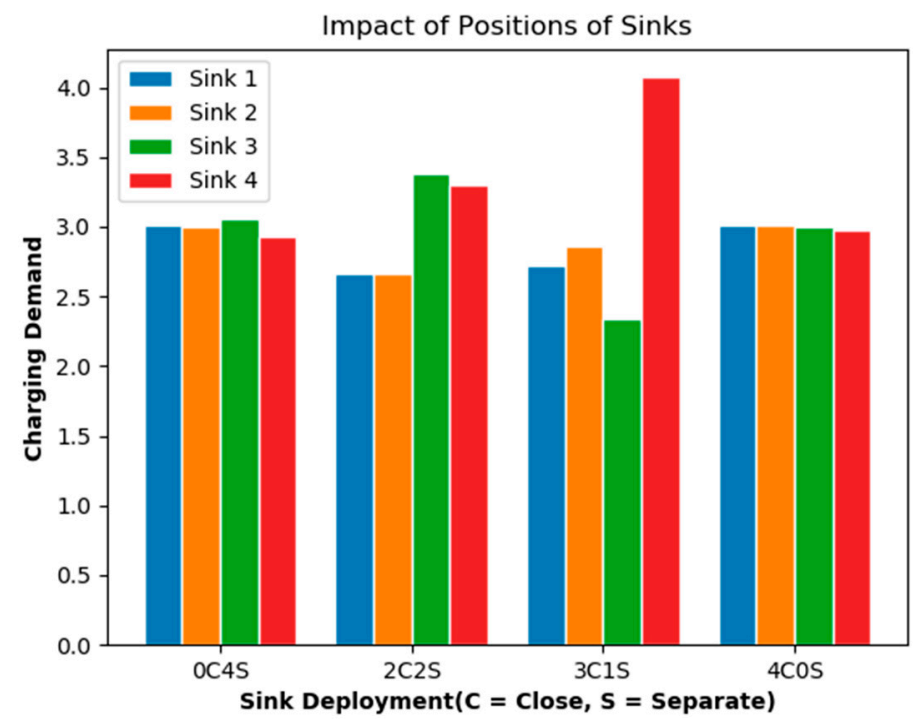

Figure 14. The impact of different densities on the energy requirements of sink nodes.

\section{Conclusions}

The deployment of WRSNs may require a large number of sensors. The use of fewer chargers in WRSNs to provide sufficient power for the sensors, reduce deployment costs, and maintain the operation of WRSNs is an important issue. Therefore, this study proposes an SMRU algorithm that uses fewer directional chargers to cover all sensors in WRSNs. In our proposed SMRU, the deployment position of the charger can be calculated in a short time, and all sensors in WRSNs are covered with a small number of chargers. Additionally, all sensor power is supplemented to avoid sensor interruption due to power consumption that causes WRSNs to fail and reduces the cost of deploying WRSNs. The performance of the proposed algorithm was compared with that of the RPRO and PBAD algorithms. Experimental results show that the average number of chargers used by the SMRU algorithm is fewer than that of RPRO and PBAD, and the execution time performance is much better than that of PBAD. In the future, we shall design a new algorithm that saves more chargers and computing time.

Author Contributions: Conceptualization, T.-L.L. and H.-Y.C.; methodology, T.-L.L. and H.-Y.C.; software, Y.-H.W.; validation, T.-L.L. and H.-Y.C.; formal analysis, T.-L.L.; investigation, T.-L.L.; resources, H.-Y.C.; writing-original draft preparation, T.-L.L. and H.-Y.C.; writing-review and editing, T.-L.L.; visualization, T.-L.L. and H.-Y.C.; funding acquisition, T.-L.L. and H.-Y.C. All authors have read and agreed to the published version of the manuscript.

Funding: This research was funded by Ministry of Science and Technology (MOST) of Taiwan, grant number 108-2321-B-415-003- and 109-2321-B-415-005-. MOST provided research funding and devices.

Conflicts of Interest: The authors declare no conflict of interest.

\section{References}

1. Pantazis, N.A.; Nikolidakis, S.A.; Vergados, D.D. Energy-Efficient Routing Protocols in Wireless Sensor Networks: A Survey. IEEE Commun. Surv. Tutor. 2013, 15, 551-591. [CrossRef]

2. Kausar, A.S.M.Z.; Reza, A.W.; Saleh, M.U.; Ramiah, H. Energy-Harvesting wireless sensor networks. Renew. Sustain. Energy Rev. 2014, 38, 973-989. [CrossRef]

3. Sample, A.P.; Meyer, D.T.; Smith, J.R. Analysis, Experimental Results, and Range Adaptation of Magnetically Coupled Resonators for Wireless Power Transfer. IEEE Trans. Ind. Electron. 2010, 58, 544-554. [CrossRef]

4. Global Wireless Charging Market 2016-2020. 2015. Available online: http://www.prnewswire.com/newsreleases/global-wireless-charging-market-2016-2020-300196623.html (accessed on 31 July 2016).

5. Gilbert, D. WiFi Routers Can Wirelessly Charge Batteries, Cameras and Sensors. 2015. Available online: http://www.ibtimes.co.uk/wifi-routers-can-wirelessly-charge-batteries-cameras-sensors-1504987 (accessed on 31 July 2016). 
6. Sendra, S.; Lloret, J.; Garcia, M.; Toledo, J.F. Power Saving and Energy Optimization Techniques for Wireless Sensor Neworks. J. Commun. 2011, 6, 439-459. [CrossRef]

7. Liao, J.-H. Wireless Charger Deployment Optimization for Wireless Rechargeable Sensor Networks. In Proceedings of the 2014 7th International Conference on Ubi-Media Computing and Workshops, Ulaanbaatar, Mongolia, 12-14 July 2013.

8. Horster, E.; Lienhart, R. Approximating optimal visual sensor placement. In Proceedings of the 2006 IEEE International Conference on Multimedia and Expo, Toronto, Canada, 9-12 July 2006.

9. Ai, J.; Abouzeid, A.A. Coverage by directional sensors in randomly deployed wireless sensor networks. J. Comb. Optim. 2006, 11, 21-41. [CrossRef]

10. Lin, T.L.; Li, S.L.; Chang, H.Y.J.E. A power balance aware wireless charger deployment method for complete coverage in wireless rechargeable sensor networks. Energies 2016, 9, 695. [CrossRef]

11. Dai, H.; Liu, Y.; Chen, G.; Wu, X.; He, T.; Liu, A.X.; Ma, H. Safe charging for wireless power transfer. Tech. Rep. 2017, 25, 3531-3544. [CrossRef]

12. He, S.; Chen, J.; Jiang, F.; Yau, D.K.Y.; Xing, G.; Sun, Y. Energy provisioning in wireless rechargeable sensor networks. IEEE Trans. Mob. Comput. 2013, 2, 1931-1942. [CrossRef]

13. Chiu, T.C.; Shih, Y.Y.; Pang, A.C.; Jeng, J.Y.; Hsiu, P.C. Mobility-Aware charger deployment for wireless rechargeable sensor networks. In Proceedings of the 2012 14th Asia-Pacific Network Operations and Management Symposium (APNOMS), Seoul, Korea, 25-27 September 2012.

14. Han, X.; Cao, X.; Lloyd, E.L.; Shen, C.C. Deploying directional sensor networks with guaranteed connectivity and coverage. In Proceedings of the 2008 5th Annual IEEE Communications Society Conference on Sensor, Mesh and Ad Hoc Communications and Networks, San Francisco, CA, USA, 16-20 June 2008.

15. Garey, M.R.; Johnson, D.S. Computers and Intractibility: A Guide to the Theory of NP-Completeness; W. H. Freeman \& Co.: New York, NY, USA, 1979.

16. Mo, L.; Kritikakou, A.; He, S. Energy-Aware Multiple Mobile Chargers Coordination for Wireless Rechargeable Sensor Networks. IEEE Internet Things J. 2019, 6, 8202-8214. [CrossRef]

17. Tang, L.; Guo, H.; Wu, R.; Fan, B. Adaptive Dual-Mode Routing-Based Mobile Data Gathering Algorithm in Rechargeable Wireless Sensor Networks for Internet of Things. Appl. Sci. 2020, 10, 1821. [CrossRef]

18. Chen, J.; Yu, C.W.; Ouyang, W. Efficient Wireless Charging Pad Deployment in Wireless Rechargeable Sensor Networks. IEEE Access 2020, 8, 39056-39077. [CrossRef]

19. Sumi, F.; Dutta, L.; Sarker, F. Future with Wireless Power Transfer Technology. J. Electr. Electron. Syst. 2018, 7, 2332-0796.1000279.

(C) 2020 by the authors. Licensee MDPI, Basel, Switzerland. This article is an open access article distributed under the terms and conditions of the Creative Commons Attribution (CC BY) license (http://creativecommons.org/licenses/by/4.0/). 